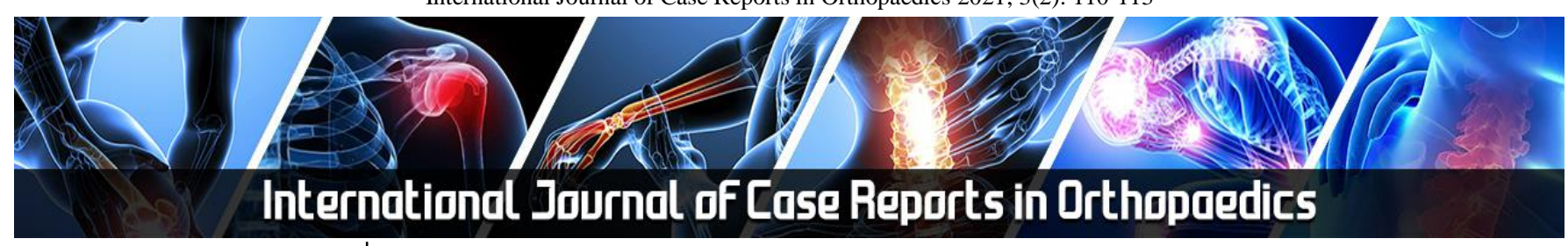

E-ISSN: 2707-8353 P-ISSN: 2707-8345 IJCRO 2021; 3(2): 110-113 Received: 02-05-2021 Accepted: 13-06-2021

Zuhdi O Elifranji MD, Department of Special Surgery, Division of Orthopaedics, School of Medicine, The University of Jordan, Amman, Jordan

Batool Almasri MD, Department of Special Surgery, Division of Orthopaedics, School of Medicine, The University of Jordan, Amman, Jordan

Shahd Alqudah MD, Department of Special Surgery, Division of Orthopaedics, School of Medicine, The University of Jordan, Amman, Jordan

Ragad sa'ed

MD, Department of Special Surgery, Division of

Orthopaedics, School of Medicine, The University of Jordan, Amman, Jordan

Alaa Albandi

MD, Department of Special Surgery, Division of Orthopaedics, School of Medicine, The University of Jordan, Amman, Jordan

Salsabil Flefil MD, Department of Special Surgery, Division of Orthopaedics, School of Medicine, The University of Jordan, Amman, Jordan

Jihad Al-Ajlouni MD, FRCS, Department of Special Surgery, Division of Orthopaedics, School of Medicine, The University of Jordan, Amman, Jordan

Faris AlShammas MD, Department of Pathology, Microbiology and Forensic Medicine, School of Medicine, The University of Jordan, Amman, Jordan

Corresponding Author: Zuhdi O Elifranji MD, Department of Special Surgery, Division of Orthopaedics, School of Medicine, The University of Jordan, Amman, Jordan

\section{Unusual orthopedic presentation of tuberculosis: A case report and review of literature}

\author{
Zuhdi O Elifranji, Batool Almasri, Shahd Alqudah, Ragad sa'ed, Alaa \\ Albandi, Salsabil Flefil, Jihad Al-Ajlouni and Faris AIShammas
}

DOI: https://doi.org/10.22271/27078345.2021.v3.i2c.79

\begin{abstract}
The case of a 77-year-old male man with right hip tuberculosis. Biopsy and aspirate were taken during debridement surgery and sent for histopathology, which confirmed the Mycobacterium tuberculosis microorganism. The patient was treated with debridement and started antimicrobial treatment.
\end{abstract}

Keywords: Osteoarticular TB, hip, tuberculosis, hip TB

\section{Introduction}

(According to the WHO classification criteria, extra pulmonary tuberculosis is defined as Mycobacterium tuberculosis infection, affecting tissues and organs outside the lung parenchyma. The EPTB cases account for $20 \%$ to $25 \%$ of all tuberculosis cases ${ }^{[1]}$. Based on published series, osteoarticular tuberculosis represents $11 \%$ of extra pulmonary tuberculosis [2].

Tuberculous arthritis can occur in almost any joint, but it often occurs in the hip or knee. It manifests as swelling, pain, and loss of joint function, and progresses in weeks to several months. Patients who develop late in the course generally have evidence of joint destruction, including local deformities and restricted range of motion. Fistula formation is common in late-stage cases. Symptoms of acute inflammation are rare. Although the smear test is less sensitive to positive cultures appears in up to $79 \%$ of cases. If culture is negative, a synovial biopsy may be required ${ }^{[3,4]}$.

The diagnosis of skeletal tuberculosis requires CT-guided biopsy for follow-up culture and pathological studies, However, a clear diagnosis requires Mycobacterium tuberculosis testing. acid-fast bacilli stains, such as ZiehlNelsen and auramine stain, enable rapid diagnosis. However, the number of samples to be detected by these stains is between 5,000 and 10,000 bacilli per milliliter ${ }^{[4]}$.

Treatment of extrapulmonary forms of tuberculosis is no different from treatment options for tuberculosis. The evidence regarding the duration of certain forms of EPTB treatment is inconsistent. The same antibacterial treatment regimen has been recommended for 6 months and prolonged treatment (12 months) is provided for central nervous system involvement ${ }^{[3]}$. Treatment options include taking rifampin, isoniazid, pyrazinamide, and ethambutol for 2 months, and then taking rifampin and isoniazid for 4 months. Once sensitivity to standard first-line drugs is determined, ethambutol can be discontinued ${ }^{[5]}$.

\section{Clinical case}

In this case report, we present a case of 77 years old male patient, not known to have any medical illnesses, presented to the clinic for the treatment of longstanding right hip pain related to known chondromatosis as shown in pelvis x-ray (figure 1) which was treated conservatively with painkillers, the pain increased in the last 3 months, it was gradual, aggravated on weight-bearing and walking, extended to the right lower extremity with restriction of movements especially abduction and external rotation of the right hip, it was very severe 10/10. The patient denied systemic symptoms such as fever, weight loss, and loss of appetite. There was no history of trauma.

After admission to a University Hospital, a battery of tests and images were conducted; blood work, microbiological assessment, chest and spine X-rays, pelvis CT scans, pelvis MRI and bone scan. The routine blood work at admission showed an elevated Erythrocytic Sedimentation Rate (ESR $96 \mathrm{~mm} / \mathrm{hr}$ ) and bacteria were seen in the urine. 
Pelvis MRI with contrast was done and compared with previous images, showing loss of joint space at the right hip joint with resorption of the articular surfaces and hypointense signal likely related to known osteochondromatosis. There were peripherally enhancing destructive changes at the right proximal femur and the right iliac bone with extraosseous fluid collections, soft-tissue swelling, and abscess formation. (Figure 2).

The patient underwent right septic hip open drainage and debridement, culture specimens were obtained and Ultrasound-Guided needle hip-joint aspiration was performed, tissue samples were sent for staining for acidfast bacilli, microscopy, culture, and sensitivities before an empiric antibiotic regime was started. The histopathological reports from the surgically extricated tissue and pus showed a granulomatous inflammatory tissue with strains of acidfast bacilli and positive M. tuberculosis PCR which was confirmed as M. tuberculosis bacilli by the microbiological testing (Figure 4).

Bone scan study was performed after good intravenous hydration and an injection of $15 \mathrm{mCi}$ 99mTc-MDP intravenously, showing an area of heterogeneous abnormal uptake in the right hip region consistent with the lytic lesion on CT and MRI.

To rule out pulmonary TB, a chest CT scan was ordered, it showed multiple mediastinal, hilar and porta hepatic calcified lymph nodes, there are also numerous and hepatic small calcified granulomas all indicative of previous (inactive) TB (Figure 3).

Antituberculous therapy (rifampicin, isoniazid, pyrazinamide) was introduced for 9 months and no recurrence was observed after a follow-up.

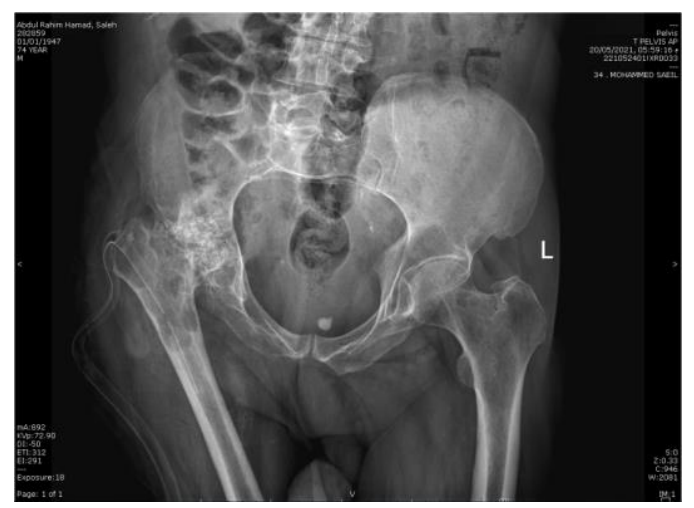

Fig 1: Pelvis x-ray

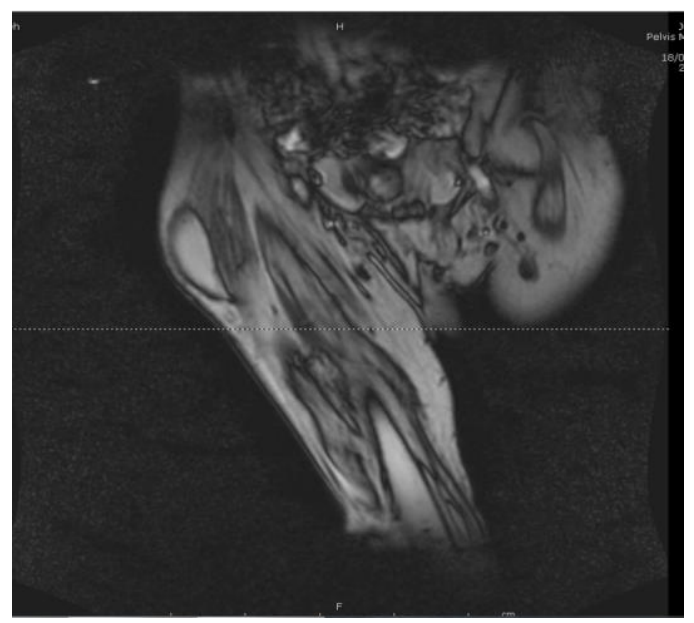

Fig 2: Pelvis MRI with contrast

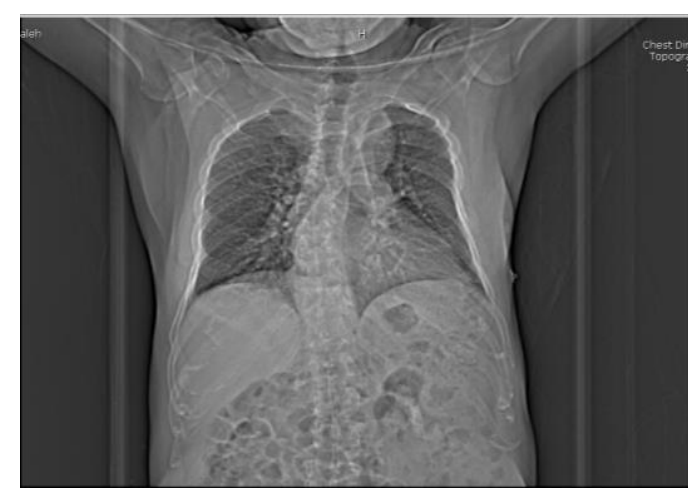

Fig 3: Chest Ct scan with iv contrast

Fig 4: Histological examination

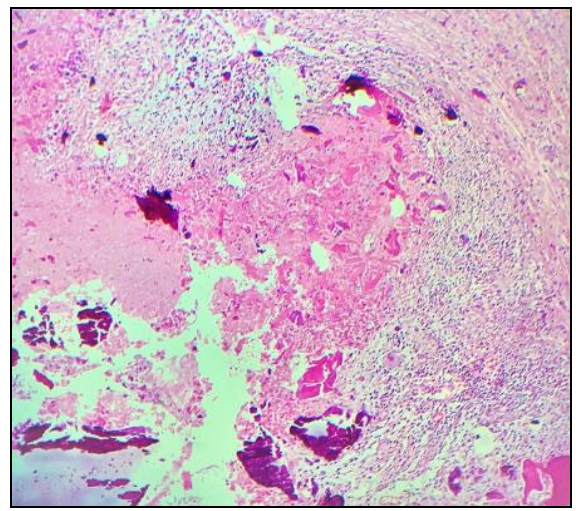

Fig 4/A: Histologic examination revealed caseating granulomas composed of an area of caseation (necrosis) surrounded by a mixed inflammatory reaction in a background of fibrous tissue and foci of calcifications (Hematoxylin \& Eosin, 10X).

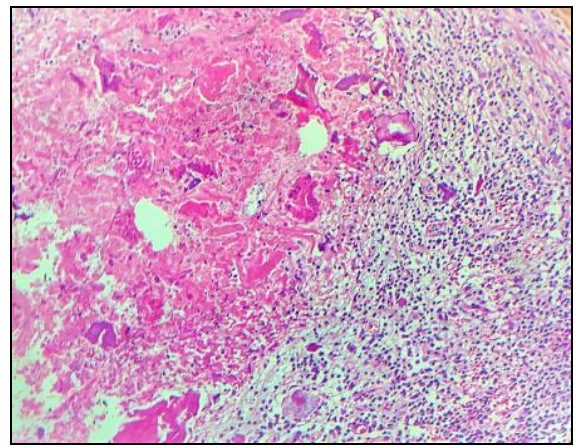

Fig 4/B: Histologic examination revealed caseating granulomas composed of an area of necrosis (left) surrounded by a mixed inflammatory reaction (right) (Hematoxylin \& Eosin, 20X).

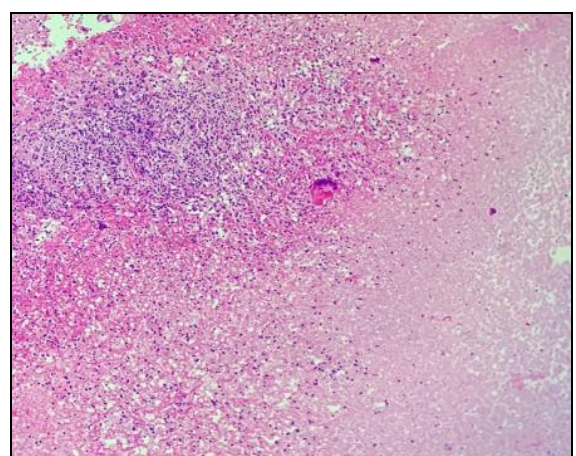

Fig 4/C: Another caseating granuloma with an area of necrosis (right side) and an adjacent mixed inflammatory reaction (left). A Langhans giant cell can be seen in the middle of this picture (Hematoxylin \& Eosin, 10X). 


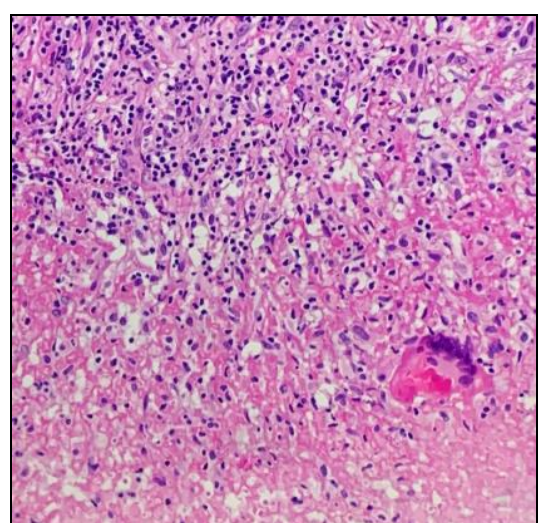

Fig 4/D: The mixed inflammatory reaction here is composed of lymphocytes, plasma cells and macrophages along with a Langhans giant cell above the area of caseation (bottom right) (Hematoxylin \& Eosin, 40X).

\section{Discussion}

Hip tuberculosis accounts for around 15-20\% of all osteoarticular tuberculosis cases. It can affect people of all ages, but it is most common in the second and third decades of life ${ }^{[6,7]}$ Because of the ambiguity of symptoms, diagnosis is frequently delayed, putting the joints at high risk of degeneration and deformity.

Osteoarticular tuberculosis can develop through hematogenous dissemination from a primary or latent focus, from lymphatic drainage or a contiguous focus of infection. Hip tuberculosis can manifest as a synovial or osseous condition. The latter may be either intraarticular or extraarticular. It is usually secondary to tuberculous osteomyelitis in which the initial site of infection is in the metaphysis due to its high blood supply, invading the end arteries causing epiphyseal bone destruction and transphyseal bacterial spread into the joint space. If treatment is delayed, cold abscesses form, which may rupture to form sinus tracts ${ }^{[8-10]}$.

Presentation depends on the severity and stage of the disease with the majority of patients complaining only from pain and swelling of the affected joint ${ }^{[11]}$. Other symptoms may include systemic symptoms of tuberculosis like night sweats and low-grade fever ${ }^{[2]}$. On physical examination, different findings may be apparent depending on the stage of the disease. In the synovitis stage, in an attempt to adjust to the excess of fluid, the joint is flexed, abducted, externally rotated and lengthened. With pain on attempted movement. In the early arthritis stage, the joint is flexed, adducted, internally rotated with noticeable shortening and mild limitation of movement. In the active stage of arthritis, hip movements become grossly restricted and are extremely painful with true shortening of the limb. In advanced arthritis, fixed deformity with marked shortening and possible dislocation and subluxation of the hip can be seen [6-7].

On radiographs, as the disease stage progresses, joint destruction increases and joint space narrowing is more prominent. Phemister's triad is characteristic of tuberculous arthritis, which includes para articular osteoporosis, peripheral erosions, and gradual narrowing of the joint space ${ }^{[10]}$. In the early stages of the disease, radiological changes may not be evident so confirmation is needed by synovial biopsy and mycobacterial culture ${ }^{[10]}$.

The differential diagnosis of hip joint tuberculosis includes subacute or chronic infections caused by other pathogens which are either bacterial such as, Staphylococcus aureus, brucella, B. pseudomallei, and Actinomyces israelii, or fungal-like candida, and Histoplasma. Metastatic malignancy may be considered especially if there are multifocal lesions ${ }^{[12]}$. In addition to that, transitory tenosynovitis, rheumatoid arthritis or osteonecrosis processes are possible differentials ${ }^{[13]}$.

Antimicrobial chemotherapy is the mainstay of treatment of hip tuberculosis. The choice of agents and the duration of treatment should be adjusted according to drug resistance, concurrent medications, and the extent of the disease ${ }^{[14]}$. In most cases receiving first-line agents (Isoniazid, rifampicin, ethambutol, pyrazinamide) duration of therapy is six to nine months. A longer duration ( 9 to 12 months) is needed when rifampin is not included in the regimen or if the disease is extensive ${ }^{[5]}$. Alongside with that, conservative non-surgical measures should be applied through the application of bilateral traction for the sake of ensuring complete hip rest and preventing deformity, followed by joint mobilization usually after 3 to 4 weeks when the pain is tolerable, the muscle spasm is less, and control on the deformity at the hip is made. Active movements of flexion, abduction and external rotation of the affected hip are encouraged for five minutes every hour at the beginning and this can be progressively increased. 7 to 10 months can be a sufficient period for achieving full weight-bearing on the joint [7] Adjunct surgical interventions are sometimes warranted to reduce deformity and improve function. The surgical options are synovectomy and joint debridement, proximal femoral osteotomy, arthrodesis [15], and total hip replacement ${ }^{[16]}$.

Upon reviewing the literature, a 3-cases study conducted by Rim Yoon et al. in $2009^{[17]}$ on hip tuberculosis found the interval of disease to surgery ranged from 3-6 months, they were all presented with pain and limited range of motion of the involved hip. They were treated with 2 weeks of preoperative anti-tubercular medications (ATT) and a onestage total hip arthroplasty (THA), followed by a 1-year ATT, which showed success and no reactivation after 18 months to 6 years of follow up.

Another study conducted in 2010 by Neogi et al. ${ }^{\text {[18] }}$ included 12 patients with hip tuberculosis who underwent THA, one non-compliant patient developed a sinus 4 months post-op which revealed a staphylococcus aureus superinfection and underwent resection arthroplasty and continued ATT for 18 months.

Sharma et al. ${ }^{[19]}$ presented a younger case in 2017 of a 28year-old female with pain in the left hip, limp and difficulty in walking for 1 year. She was treated for 2 months with ATT preoperatively followed by a THA and ATT for 1 year, she showed clinical improvement and no reactivation at 1 year follow up.

Ramos et al., ${ }^{[20]}$ presented a case of a 3-year history of tuberculosis of the hip, she underwent drainage and resection arthroplasty with osteotomy followed by a 9 months 3 drugs course of ATT. Improved clinically with no recurrence after 1 year of follow-up.

Wang et al., ${ }^{[21]}$ reviewed 6 cases of tubercular hip arthritis, two of which had draining sinuses. They were all given 4 drugs ATT for at least 2 weeks before surgery and 12 months after. Of the two with sinuses, one had cemented and the other non-cemented THA. On the last follow-up, full ambulation was achieved, and no loose prostheses were located in all subjects. 
Jeddo et al. ${ }^{[11]}$ reviewed a patient who was treated for hip tuberculosis with ATT medications and prophylactic antibiotics, the patient later started to deteriorate gradually until diagnosed with a recurrence of hip tuberculosis after 40 years, he was given 3 drugs ATT for 3 months and underwent THA followed by 1 year ATT, he showed clinical improvement at the 2 years follow up.

In our case of a 77-year-old male patient diagnosed with tuberculous hip arthritis. His presentation was similar to the reviewed articles as hip pain and restriction of movements especially abduction and external rotation. The patient had open sinus drainage with careful surgical debridement and was given 3 anti-tubercular medications, he has shown improvement over the first 4 months with no signs of recurrence, and ATT planned to continue for 9 months of total therapy.

\section{References}

1. Meischner W. [Ernst Heinrich Weber, 1795-1878]. Z Psychol Z Angew Psychol 1978;186(2):159-69.

2. Peto HM, Pratt RH, Harrington TA, LoBue PA, Armstrong LR. Epidemiology of Extrapulmonary Tuberculosis in the United States, 1993-2006. Clinical Infectious Diseases 2009;49(9):1350-7.

3. Gale-Rowe M, Menzies D, Sutherland J, Wong T. Highlights of the new 7th edition of the Canadian Tuberculosis Standards. Can Commun Dis Rep 2014;40(6):113-6.

4. Tuli SM. General principles of osteoarticular tuberculosis. Clin Orthop Relat Res 2002(398):11-9.

5. Blumberg HM, Leonard MK, Jr., Jasmer RM. Update on the treatment of tuberculosis and latent tuberculosis infection. Jama 2005;293(22):2776-84.

6. Saraf SK, Tuli SM. Tuberculosis of hip: A current concept review. Indian J Orthop 2015;49(1):1-9.

7. Babhulkar S, Pande S. Tuberculosis of the hip. Clin Orthop Relat Res 2002(398):93-9.

8. Leonard Michael K, Blumberg Henry M, Schlossberg D. Musculoskeletal Tuberculosis. Microbiology Spectrum 2017;5(2):5.2.28.

9. De Vuyst D, Vanhoenacker F, Gielen J, Bernaerts A, De Schepper AM. Imaging features of musculoskeletal tuberculosis. Eur Radiol 2003;13(8):1809-19.

10. Griffith JF, Kumta SM, Leung PC, Cheng JCY, Chow LTC, Metreweli C. Imaging of Musculoskeletal Tuberculosis: A New Look at an Old Disease. Clinical Orthopaedics and Related Research®. 2002, 398.

11. Jeddo $\mathrm{S}$, Huang $\mathrm{CW}, \mathrm{Li} \mathrm{M}$. Case report on the recurrence of tuberculosis of hip after 40 years. SpringerPlus 2014;3(1):662.

12. Ye M, Huang J, Wang J, Ren J, Tu J, You W et al. Multifocal musculoskeletal tuberculosis mimicking multiple bone metastases: a case report. BMC Infect Dis 2016;16:34.

13. Paño JR, Pigrau C, Morte E, Almirante B, Muñoz P, Bouza E. A man from Morocco and chronic hip pain. Rev Esp Quimioter 2019;32(1):78-82.

14. Nahid P, Dorman SE, Alipanah N, Barry PM, Brozek JL, Cattamanchi A et al. Official American Thoracic Society/Centers for Disease Control and Prevention/Infectious Diseases Society of America Clinical Practice Guidelines: Treatment of DrugSusceptible Tuberculosis. Clin Infect Dis 2016;63(7):e147-e95.
15. Jain AK. Tuberculosis of the skeletal system. Indian $\mathbf{J}$ Orthop 2016;50(3):337.

16. Tiwari A, Karkhur Y, Maini L. Total hip replacement in tuberculosis of hip: A systematic review. J Clin Orthop Trauma 2018;9(1):54-7.

17. Yoon TR, Rowe SM, Anwar IB, Chung JY. Active tuberculosis of the hip treated with early total hip replacement--a report of 3 cases. Acta Orthopaedica Scandinavica 2001;72(4):419-21.

18. Neogi DS, Yadav CS, Kumar A, Khan SA, Rastogi S. Total Hip Arthroplasty in Patients with Active Tuberculosis of the Hip with Advanced Arthritis. Clinical Orthopaedics and Related Research®. 2010, 468(2).

19. Sharma H, Dreghorn CR, Gardner ER. Girdlestone resection arthroplasty of the hip: Current perspectives. Current Orthopaedics 2005;19(5):385-92.

20. Ramos CG, Alves MD, Santos RPd, Falci D, Goldani LZ. Chronic slowly progressive monoarthritis tuberculosis of the hip without systemic symptoms mimicking osteoarthritis: a case report. Cases Journal 2009;2(1):6457.

21. Wang Y, Wang J, Xu Z, Li Y, Wang H. Total hip arthroplasty for active tuberculosis of the hip. International Orthopaedics 2010;34(8):1111-4. 